\title{
Multiple-Sourcing-Strategien bei Finanzdienstleistern - Eine Analyse zum Einfluss der Integrationskosten am Beispiel der Wertpapierabwicklung
}
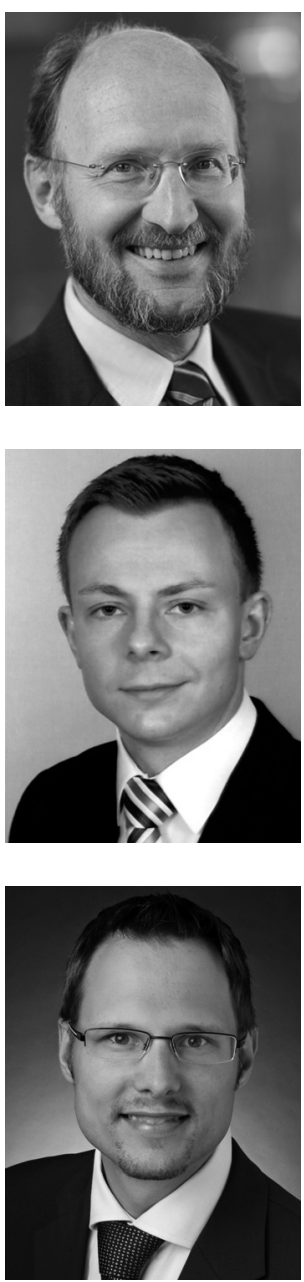

\section{Hans Ulrich Bubl, Christoph Dorsch und Matthias Henneberger}

Finanzdienstleister, Informations- und Kommunikationssysteme, Multiple-Sourcing, Optimierungsmodell, Risikosteuerung, SupplyChain-Management

Financial service providers, information and communication systems, multiple sourcing, economic model, risk management, supply chain management

Während Multiple-Sourcing-Strategien zur Steuerung von Ausfallrisiken in der Industrie bereits gängige Praxis sind, spielen sie bei Finanzdienstleistern auch bei zeitkritischen Transaktionen bisher keine Rolle. Grund hierfür sind die hohen Kosten für die technische Integration von Dienstleistern. Unter der Annahme, dass diese Integrationskosten durch Standardisierung und Flexibilisierung sinken, wird am Beispiel der Wertpapierabwicklung mit einem Optimierungsmodell ein beim Multiple-Sourcing zu berücksichtigender Zielkonflikt zwischen Integrationskosten und Risikokosten durch mögliche Ausfälle betrachtet. Zudem wird untersucht, wie sich Multiple-Sourcing auf die Anforderungen an die notwendige Ausfallsicherheit der Dienstleister auswirkt. Es zeigt sich, dass bei Finanzdienstleistern Multiple-Sourcing ökonomisch sinnvoll sein kann und damit auch die derzeit hohen Anforderungen an die Ausfallsicherheit reduziert werden können.

While multiple sourcing is common practice in manufacturing to mitigate risk of supplier breakdown, those strategies are hardly ever applied by financial service providers, not even for time-critical transactions. This is due to the high costs for technical integration of service providers. Assuming a decrease in these costs as a consequence of standardization and an improvement of flexibility, this paper analyzes the economic benefits of multiple sourcing for the financial services industry. Drawing on the example of securities settlement the trade-off between the costs of integration and the costs of risk due to service provider breakdown is considered in an economic model. Moreover, we analyze the implications of multiple sourcing on availability requirements. The analysis reveals that multiple sourcing for financial service providers 
can be reasonable from an economic point of view and therefore the requirements on availability of the service provider base can be reduced.

\section{Ausgangssituation und Forschungsfragen}

Sourcing-Fragestellungen gewinnen bei Finanzdienstleistern (FDL) weiter an Bedeutung. Traditionell hoch integrierte Banken konzentrieren sich auf Kernkompetenzen in Beratung und Vertrieb und lagern andere Teile ihrer Wertschöpfung an spezialisierte Dienstleister aus (Falkenberg et al. 2006; Alt et al. 2009). Dies gilt auch für zeitkritische Prozesse wie die Abwicklung von Wertpapiergeschäften (Mansfeld et al. 2010). Eine Transaktionsbank übernimmt dabei als Dienstleister u. a. die Weiterleitung einer Order an den Handelsplatz, die Verrechnung des Geschäfts mit den beteiligten Institutionen, das Reporting und die Verwahrung des Wertpapiers (Kröpfl 2003). Mit diesen zeitkritischen Prozessen sind hohe operationelle Risiken verbunden, z. B. aus dem Ausfall des Dienstleisters und der damit verbundenen Verzögerung bei der Bearbeitung. Daher werden heute hohe Verfügbarkeitsanforderungen an die Dienstleister gestellt. Dagegen spielen Multiple-Sourcing-Strategien, also der Bezug einer Dienstleistung von mehr als einem Anbieter, wie sie in der Industrie bereits gängige Praxis sind, in der FDL-Branche bisher nur eine untergeordnete Rolle (Craighead et al. 2007; Regniet 2007).

Ein Grund für den Verzicht auf Multiple-Sourcing sind die vor dem Aufkommen neuerer Integrationskonzepte Ende der 1990er Jahre vorherrschenden, sehr hohen Kosten für die technische Integration der Dienstleister (Joiko 2006; Alt/Zerndt 2008). ${ }^{1}$ Diese spielen gerade bei Wertpapiertransaktionen, wo es v. a. um die automatisierte Weitergabe und Verarbeitung von Informationen geht, als Teil der Transaktionskosten eine herausragende Rolle. So muss der Dienstleister bei der Wertpapierabwicklung eng an die Anwendungssysteme (AWS) des FDL angebunden werden, z. B. für den Zugriff auf die Stammdaten der Kunden, die Orderübermittlung, das Meldewesen oder das Risikomanagement (Weitzel et al. 2003). Erschwerend wirken die in der Branche vorherrschenden, komplexen AWSLandschaften mit unterschiedlichsten, meist veralteten Eigenentwicklungen (Sterling Commerce 2010).

Neue Integrationskonzepte und -technologien haben in den letzten Jahren jedoch zunehmend an Reife gewonnen und Verbreitung in der praktischen Anwendung gefunden. Derzeit wird v. a. das Konzept der Serviceorientierten Architekturen (SOA) diskutiert (Harishankar 2009). Geschäftsprozesse werden dabei aus einzelnen, in sich geschlossenen und mit standardisierten Schnittstellen und Datenaustauschformaten arbeitenden Services kombiniert. Ein Service stellt jeweils die für eine oder mehrere Aktivitäten des Geschäftsprozesses benötigte Funktionalität bereit. SOA ermöglichen u. a. eine schnelle Rekonfiguration bei Änderungen an dem zu unterstützenden Geschäftsprozess und erhöhen damit die Flexibilität. Gleichzeitig wird es durch den modularen Aufbau und die Standardisierung einfacher, ganze Prozesse oder auch nur einzelne Aktivitäten an Dienstleister auszulagern (Erl 2007). Ein weiterer Vorteil für die Integration von Dienstleistern ergibt sich aus

1 Zeitlich mit dem Aufkommen neuer Integrationskonzepte erstellte Studien u. a. der Gartner Group zeigen, dass $35 \%$ bis $60 \%$ der IT-Budgets zur Integration von Anwendungssystemen aufgewendet wurden (Rub et al. 2001). Eine Schnittstelle zwischen zu integrierenden Anwendungssystemen konnte laut Aberdeen Group Kosten von bis zu 10 Millionen US-Dollar verursachen (Linthicum 2003). 
den der Einführung von SOA oft vorgelagerten Projekten zur Standardisierung und Optimierung zu unterstützender Geschäftsprozesse (Becker et al. 2009).

Da FDL eine Vorreiterrolle bei der Anwendung dieser neuen Technologien und Standards einnehmen, ${ }^{2}$ ist zu erwarten, dass v. a. technische Integrationskosten bei der Anbindung von Dienstleistern stark sinken. Ob daher auch dort Multiple-Sourcing-Strategien ${ }^{3}$ ökonomisch sinnvoll eingesetzt werden können, um die mit Ausfällen verbundenen Kostenwirkungen zu verringern (im Folgenden kurz: Risikosteuerung), ist Gegenstand dieses Beitrags:

Kann Multiple-Sourcing vor dem Hintergrund sinkender technischer Integrationskosten zur Risikosteuerung in Wertschöpfungsnetzen von FDL ökonomisch sinnvoll sein?

Wenn durch den ökonomisch sinnvollen Einsatz von Multiple-Sourcing die Risikokosten eines Ausfalls verringert werden können, wird damit jedoch auch die bisherige Strategie der FDL in Frage gestellt, Ausfälle über hohe Anforderungen an die Verfügbarkeit technischer Systeme zu reduzieren. Dies führt zu einer Erweiterung der Fragestellung:

Wie wirkt sich Multiple-Sourcing auf die Anforderungen an die Verfügbarkeit der technischen Systeme und damit auf die Summe aus Risikokosten eines Ausfalls und den Kosten für Ausfallsicherheitsmassnahmen als relevante Entscheidungsgrösse aus?

Zur Beantwortung der Forschungsfragen und Ableitung von Gestaltungsempfehlungen für robuste Wertschöpfungsnetze bei FDL wird im Folgenden ein Optimierungsmodell entwickelt, welches den diskutierten Zielkonflikt zwischen Integrationskosten, Risikokosten eines Ausfalls ${ }^{4}$ und Kosten für Ausfallsicherheitsmassnahmen technischer Systeme abbildet.

\section{Literaturüberblick}

Den Zielkonflikt von Integrations- und Risikokosten bzw. die Fragestellung nach der Anzahl anzubindender Dienstleister behandeln die folgenden Veröffentlichungen:

Homburg (1995) stellt ein Modell vor, welches den Trade-Off zwischen den von der Anzahl der Lieferanten abhängigen Transaktionskosten und den Risikokosten des Ausfalls eines oder mehrerer Lieferanten betrachtet. Optimiert wird nach der kostenminimalen Anzahl von Lieferanten. Aus der Modellanalyse werden Hypothesen abgeleitet, welche anschliessend einer empirischen Untersuchung unterzogen werden. Die Hypothesen, z. B. über den Einfluss der Komplexität des vom Lieferanten bereitgestellten Produkts oder der Anzahl am Markt agierender Lieferanten, wurden dabei weitgehend empirisch bestätigt. Die optimale Anzahl an Lieferanten ermitteln auch Berger et al. (Berger et al. 2004; Berger/Zeng 2006). Sie untersuchen den Trade-Off zwischen Transaktions- und Risikokosten des Ausfalls eines oder aller Lieferanten anhand eines Entscheidungsbaumes. Dieser Ansatz wird von Ruiz-Torres/Mabmoodi (2007) erweitert, indem zusätzlich die Möglichkeit

2 Bereits 2006 ersetzten ca. drei Viertel der befragten europäischen FDL vor allem Kernbank-Systeme, z. B. zur Abwicklung von Zahlungs- und Wertpapiertransaktionen, wobei zunehmend Standardsoftware verwendet (Quack 2010) und SOA (Friedrich 2006) eingesetzt wurden. Als wichtigsten Grund für die unverändert hohe Investitionsbereitschaft und -notwendigkeit wird die erleichterte Disaggregation der Wertschöpfungskette genannt (Weitzel et al. 2003; Wortmann 2006).

3 Vor- bzw. nachgelagerte Outsourcing-Entscheidungen, z. B. welche Teile der Leistungserstellung überhaupt an Dienstleister ausgelagert werden sollen, und damit verbundene Kosten sind nicht Gegenstand dieses Beitrags.

4 Ausfälle, welche sich durch technische Ausfallsicherheitsmassnahmen nicht beeinflussen lassen (z. B. aus einer Insolvenz) sind nicht Gegenstand dieses Beitrags. 
des Ausfalls jedes einzelnen Lieferanten berücksichtigt wird. Die Ergebnisse werden jeweils einer Sensitivitätsanalyse unterzogen und dabei u. a. untersucht, inwieweit sich das Verhältnis von Transaktions- und Risikokosten oder die Ausfallwahrscheinlichkeit der Lieferanten auswirkt. Wang/Zhao (2007) erweitern diesen Ansatz wiederum, indem die Kapazität aller verfügbaren Lieferanten Berücksichtigung findet. Die Sensitivitätsanalyse zeigt, dass v. a. die Zuverlässigkeit der Lieferanten das Optimum beeinflusst, wenn die Kapazität der Lieferanten die Gesamtnachfrage aller Nachfrager übersteigt. Sind hingegen Gesamtnachfrage und Gesamtkapazität gleich, wird das Optimum hauptsächlich vom Verhältnis der Transaktions- zu den Risikokosten beeinflusst.

Bislang nicht betrachtet wurden die in diesem Beitrag adressierten Forschungsfragen, welche sich einer Besonderheit im Kernbereich der FDL-Branche widmen: Dienstleistungen sind dort v. a. durch die hochautomatisierte Verarbeitung von Informationen verschiedener Quellen charakterisiert. Vor dem Hintergrund neuer Integrationskonzepte sind als Transaktionskosten neben organisatorischen, rechtlichen und laufenden Kosten also gerade die einmalig zu entrichtenden technischen Integrationskosten entscheidungsrelevant, wenn über die optimale Anzahl anzubindender Dienstleister zu entscheiden ist. Darüber hinaus ist der in diesem Beitrag betrachtete Ausfall eines Dienstleisters meist eng an die Verfügbarkeit der technischen Systeme gekoppelt, welche durch Investitionen in Ausfallsicherheitsmassnahmen beeinflusst werden kann. Da diese Investitionen zu höheren Abwicklungspreisen führen und so wieder auf den auslagernden FDL übergewälzt werden, sind Kosten für Ausfallsicherheitsmassnahmen für eine ökonomisch sinnvolle Entscheidung zu berücksichtigen und deren optimale Höhe mit zu bestimmen.

\section{Modell zur Optimierung der Dienstleisteranzahl}

Dass sich gerade die Wertpapierabwicklung für Multiple-Sourcing eignet, zeigt deren Untersuchung auf Netzwerkfähigkeit, also die „[...] Fähigkeit zur schnellen und effizienten Bildung, Durchführung und Weiterentwicklung von IT-gestützten Geschäftsbeziehungen“ (Alt/Fleisch 2002, 356). Netzwerkfähigkeit lässt sich auf verschiedenen Gestaltungsebenen betrachten und verbessern. So sind netzwerkfähige Leistungen und Prozesse durch Standardisierung und Digitalisierung zu erreichen. Netzwerkfähige Informationssysteme zeichnen sich v. a. durch eine durchgängige Integration sowie Kommunikations- und Datenstandards aus. Des Weiteren tragen flexible Organisationsstrukturen sowie eine kooperationsfördernde Unternehmenskultur mit entsprechend befähigten Mitarbeitern wesentlich zur Netzwerkfähigkeit bei.

Die Netzwerkfähigkeit der Leistung ist bei der Wertpapierabwicklung sehr positiv zu bewerten. Zum einen ist sie bereits heute weitgehend digitalisiert. Zum anderen haben sich durch die notwendige Abstimmung mit den vielen beteiligten Partnern und die gesetzlichen Vorschriften sehr standardisierte Leistungen herausgebildet, bei welchen eine Individualisierung keinen wesentlichen Wettbewerbsvorteil bietet. Auf den Ebenen Prozesse und Informationssystem führt gerade der Einsatz neuer Integrationskonzepte zu einer wesentlichen Steigerung der Netzwerkfähigkeit. Gründe sind z. B. die mit einer Einführung von SOA oft einhergehenden Massnahmen zur Prozessoptimierung sowie die Verwendung einheitlicher Schnittstellen und Datenformate auf Ebene der Informationssysteme. Dass SOA einen positiven Einfluss auf die Netzwerkfähigkeit hat, wurde von Kohlmann/Alt (2010) empirisch bestätigt. Hinsichtlich Organisationsstruktur, Unternehmenskultur und Mitarbeitern ist festzustellen, dass auf Grund individueller Unterschiede zwischen Unternehmen 
generalisierende Aussagen zur Netzwerkfähigkeit problematisch sind. Diese Ebenen sind für jedes Unternehmen im Einzelnen zu analysieren. Tendenziell kann jedoch vermutet werden, dass der Trend zu schlanken und spezialisierten FDL, welche untereinander vernetzt die traditionelle Universalbank zunehmend ablösen sollen, die Netzwerkfähigkeit auch auf diesen Ebenen nachhaltig verbessert.

\subsection{Modellannahmen und Optimierung}

(A1) Über den Betrachtungszeitraum, welcher sich diskret in Zeiteinheiten unterteilt, sind die Kosten der Integration $K_{I}(n) \in \mathrm{IR}_{++}$von Transaktionsbanken (TB), die erwarteten Risikokosten des Ausfalls $K_{R}(n, w) \in \mathrm{IR}_{+}$einer oder mehrerer angebundener TB und die Kosten für Ausfallsicherheitsmassnahmen $K_{A}(w) \in \mathrm{IR}_{+} \mathrm{zu}$ betrachten. Somit ergeben sich die für das Modell relevanten und zu minimierenden Gesamtkosten $K_{G}(n, w) \in \mathrm{IR}_{++}$mit

$$
K_{G}(n, w)=K_{I}(n)+K_{R}(n, w)+K_{A}(w) \rightarrow \operatorname{Min} !
$$

Es ist ex ante über die optimale Anzahl $n^{*} \in \mathrm{IN}_{++}$anzubindender Transaktionsbanken zu entscheiden, welche die Gesamtkosten minimiert. Eine gleichzeitige Optimierung der Ausfallwahrscheinlichkeit kann zudem Aufschluss darüber geben, ob bzw. in welchen Fällen eine Erhöhung der Anzahl der Transaktionsbanken bei gleichzeitiger Reduzierung von Ausfallsicherheitsmassnahmen ökonomisch sinnvoll ist.

Die Integrationskosten $K_{\mathrm{I}}$ enthalten alle Kosten für die technische Integration und die Koordination der Dienstleister (z. B. Abstimmung der AWS, Vereinbarung von Service-Level, Governance-Kosten). ${ }^{5}$ Da durch neue Integrationskonzepte nur technische Integrationskosten beeinflusst werden, sind diese explizit von anderen Integrationskosten getrennt. Es ist anzunehmen, dass die Kosten insgesamt mit der Anzahl angebundener Transaktionsbanken linear bis degressiv steigen. Es können auch Kostenbestandteile existieren, die sich aufgrund zunehmender Komplexität bei mehreren Transaktionsbanken progressiv verhalten (Homburg 1995). Diese haben aber aufgrund des hohen Automatisierungsgrades in der Abwicklung einen geringen Anteil. Um beide Fälle zu berücksichtigen, wird im Modell von proportional steigenden Kosten als Grenzfall ausgegangen. Auswirkungen anderer Kostenverläufe werden beim Fallbeispiel diskutiert.

(A2) Je TB fallen Kosten an, die sich in technische Integrationskosten $k_{T} \in \mathrm{IR}_{++}$Geldeinheiten und nicht-technische Integrationskosten $k_{N} \in \mathrm{IR}_{++}$Geldeinheiten unterteilen. Die Integrationskosten $K_{\mathrm{I}}(n)$ steigen proportional mit der Anzahl anzubindender TB:

$$
K_{I}(n)=\left(k_{T}+k_{N}\right) \cdot n \text {. }
$$

Die Bestimmung der relevanten Risikokosten $K_{\mathrm{R}}$ lehnt sich an bestehende Modelle (Spabr 2001; Kröpfl 2003) an. Dabei werden die Risikokosten über die Wahrscheinlichkeit für

5 Die grundlegende Entscheidung, an eine Transaktionsbank auszulagern, ist bereits gefallen. Daher sind (allein damit einhergehende) Kosten für die erstmalige Einrichtung der Zusammenarbeit mit einem Dienstleister nicht entscheidungsrelevant. 
den Eintritt eines Ausfalls (Häufigkeit) multipliziert mit der Dauer des Ausfalls und der Höhe des verzögerten Transaktionsvolumens (Schwere) ermittelt.

(A3) Zu Beginn jeder Zeiteinheit sind $t \in \mathrm{IN}_{++}$Transaktionen (TA) abzuwickeln. Diese werden gleichmässig ${ }^{6}$ auf die zur Verfügung stehenden TB verteilt und unmittelbar abgewickelt. Jede TB kann zu jeder Zeiteinheit beliebig viele TA abwickeln. Die nicht beeinflussbare Abwicklungsdauer beträgt $b \in \mathrm{IN}_{++}$Zeiteinheiten und das durchschnittliche Volumen einer TA $v \in \mathrm{IR}_{++}$Geldeinheiten.

Die diskreten Zeiteinheiten bilden ab, dass die Abwicklung von Transaktionen derzeit meist als diskreter Tagesend- bzw. Batchlauf einmal am Tag angestossen wird. Zwar können Wertpapiergeschäfte sofort ausgeführt werden, die Abwicklung der Transaktion erfolgt jedoch verzögert und nimmt dann im Allgemeinen einige Zeit in Anspruch (Prozesszeit $b$ ).

(A4) Bei einem Ausfall einer TB entstehen Kosten durch die daraus resultierende Verzögerung der Abwicklung. Es wird der Erwartungswert der Risikokosten $K_{\mathrm{R}}$ zur Entscheidung herangezogen. Dieser berechnet sich über den Risikokostensatz $r>0$, mit welchem das jeweilige TA-Volumen je verzögerter Zeiteinheit zu gewichten ist. Der Risikokostensatz bildet alle durch die Verzögerung entstehenden (Mehr-)Kosten ab. Für das in diesem Beitrag betrachtete Risiko eines Ausfalls der Transaktionsbank gibt es vielfältige Gründe. Häufig auftretende Ausfälle durch Störungen der technischen Systeme und Kommunikationsverbindungen haben - wie selten eintretende Ereignisse mit entsprechend grösseren Auswirkungen aus Angriffen auf Datennetze, Naturkatastrophen und Anschlägen - hohes Schadenspotential und führen ggf. zu massiven Beeinträchtigungen (Berger et al. 2004; Wang/Zhao 2007). Die aktuelle Krise an den Finanzmärkten zeigt, dass auch kumuliert auftretende, längerfristige Ausfälle in der FDL-Branche nicht auszuschliessen sind. Kosten entstehen bei einem Ausfall z. B. durch notwendige manuelle Eingriffe in den automatisierten Prozess oder Schadensersatzzahlungen durch Kundenreklamationen. Zudem erhöhen bzw. verlängern sich durch die nicht fristgerechte Abwicklung der betroffenen Transaktionen Terminrisiken oder es ergeben sich Liquiditätsengpässe.

(A5) Die TB fallen unabhängig voneinander zu Beginn einer beliebigen, aber einheitlichen Zeiteinheit, jeweils mit Ausfallwahrscheinlichkeit $w \in] 0 ; 1[$ (bezogen auf den Betrachtungszeitraum) aus.

(A6) Während des Ausfalls der Dauer $d \in \mathrm{IN}_{++}$Zeiteinheiten kann die betroffene TB keine TA abwickeln. Neu eintreffende TA können ohne Zeitverzögerung und ohne zusätzliche Kosten auf andere TB umgeleitet werden, nicht jedoch TA, die sich zum Zeitpunkt des Ausfalls noch im Abwicklungsprozess bei der vom Ausfall betroffenen TB befinden.

Die von den Transaktionsbanken angebotenen Leistungen (abgesehen vom Umfang, welcher sich aus der ausgelagerten Prozesstiefe ergibt) unterscheiden sich hinsichtlich ihrer Qualität (Abwicklungsdauer, Ausfallwahrscheinlichkeit etc.) nicht wesentlich. Dies wurde bereits bei der Untersuchung der Wertpapierabwicklung auf Netzwerkfähigkeit festgehal-

6 Für das Optimierungsmodell ist der Erwartungswert der Risikokosten relevant. Dieser steigt linear mit dem vom Ausfall betroffenen Transaktionsvolumen. Da der Erwartungswert für jede denkbare Verteilung der Transaktionen auf die angebundenen Transaktionsbanken identisch ist, kann hier o. B. d. A. eine gleichmässige Aufteilung angenommen werden. 
ten: Von den FDL werden weitgehend standardisierte Leistungen angeboten, da sich diese bei der Wertpapierabwicklung kaum gegenüber ihren Kunden differenzieren können. Es handelt es sich um einen Markt von sogenannten Commodity-Produkten, also Produkten homogener Qualität. Dies begründet die zunächst sehr verallgemeinernd erscheinende Annahme identischer Eigenschaften der einzelnen Transaktionsbanken.

Die Beschränkung der Umleitung auf neu eintreffende Transaktionen trägt der Tatsache Rechnung, dass der Flexibilität in der Realität gewisse Grenzen gesetzt sind. Ist die Abwicklung einer Transaktion angestossen und sind z. B. von Externen zugeführte Informationen wie Ausführungsbestätigungen oder Lieferlisten bereits verarbeitet, erscheint aus Konsistenzgründen nur eine Rücknahme der bisher erfolgten Abwicklungsschritte und die komplette Neuabwicklung sinnvoll.

Formel (3) fasst die nun definierten Risikokosten in Abhängigkeit der Anzahl angebundener Transaktionsbanken und der Ausfallwahrscheinlichkeit zusammen (siehe Anhang):

$$
K_{R}(n, w)=\left[b \cdot\left(d+\frac{b}{2}\right) \cdot w+\frac{d^{2}}{2} \cdot w^{n}\right] \cdot t \cdot v \cdot r .
$$

Für die Kosten für Ausfallsicherheitsmassnabmen $K_{\mathrm{A}}$ sind zwei Eigenschaften evident: Einerseits wachsen diese mit sinkender Ausfallwahrscheinlichkeit c. p. überproportional an, da immer grössere Investitionen z. B. für redundante Datenleitungen getätigt werden müssen. Zum anderen kann eine Ausfallwahrscheinlichkeit von Null praktisch nie erreicht werden.

(A7) Die Kosten für Ausfallsicherheitsmassnahmen $K_{A}(w)$ folgen einer über $w$ stetigen, streng monoton fallenden und streng konvexen Kostenfunktion mit

$$
\lim _{w \rightarrow 0} K_{A}(w) \rightarrow \infty\left(\lim _{w \rightarrow 0} \frac{\partial K_{A}(w)}{\partial w} \rightarrow 0\right) \text { und } \lim _{w \rightarrow 1} K_{A}(w)=\lim _{w \rightarrow 1} \frac{\partial K_{A}(w)}{\partial w}=0 .
$$

Es ist

$$
K_{A}(w)=(-\ln w) \cdot k_{A} \text { mit } k_{A} \in \mathrm{IR}_{++}
$$

eine mögliche Ausprägung der Kostenfunktion für Ausfallsicherheitsmassnahmen, welche die genannten Eigenschaften erfüllt und über den Kostenparameter $k_{A}$ skalierbar ist. Sie konkretisiert $K_{A}(w)$ zur weiteren Modellanalyse.

Die Gesamtkosten $K_{G}(n)$ ergeben sich nun nach Annahme (A1) zu:

$$
K_{G}(n, w)=\left(k_{T}+k_{N}\right) \cdot n+\left[b \cdot\left(d+\frac{b}{2}\right) \cdot w+\frac{d^{2}}{2} \cdot w^{n}\right] \cdot t \cdot v \cdot r+(-\ln w) \cdot k_{A} \rightarrow \operatorname{Min} !
$$

Um die grundlegende Forschungsfrage nach dem ökonomisch sinnvollen Einsatz von Multiple-Sourcing zu beantworten, wird die Gesamtkostenfunktion zunächst nur nach dem 
Parameter $n$ optimiert und dazu als stetige Variable $n \in \operatorname{IR}$ angenommen. ${ }^{7} K_{G}(n)$ ist streng konvex und besitzt ein globales Minimum (siehe Anhang) bei

$$
\dot{n}^{*}=\ln \left(-\frac{2 \cdot\left(k_{T}+k_{N}\right)}{d^{2} \cdot t \cdot \ln w \cdot V \cdot r}\right) \cdot(\ln w)^{-1}
$$

woraus sich im Hinblick auf die grundlegende Forschungsfrage mit

$$
k_{T}<\frac{d^{2} \cdot t \cdot v \cdot r \cdot(-\ln w) \cdot w}{2}-k_{N}
$$

die Bedingung herleiten lässt, für welche $\dot{n}^{*}>1$ gilt. Steigende Ausfallwahrscheinlichkeiten führen dabei c. p. zunächst erwartungsgemäss zu steigendem $\dot{n}^{*}$. Bei hohen Ausfallwahrscheinlichkeiten kehrt sich dies jedoch um, da die dadurch verursachte Steigerung der Gesamtkosten nicht mehr durch Hinzunahme einer weiteren Transaktionsbank kompensiert werden kann. Dies ist insbesondere im Hinblick auf die erweiterte Forschungsfrage interessant, zu deren Beantwortung eine gleichzeitige Optimierung nach der Anzahl anzubindender Transaktionsbanken und der Ausfallwahrscheinlichkeit notwendig ist.

Im folgenden Abschnitt wird sowohl das o. a. Ergebnis der Optimierung mit einem Fallbeispiel verdeutlich und weiter diskutiert, wie auch die erweiterte Forschungsfrage aufgegriffen. Diese wird daran anschliessend anhand einer computergestützten Simulation näher untersucht. Die dabei gewonnen Ergebnisse sollen Aussagen auch über die konkrete Parameterkonstellation im Fallbeispiel hinaus ermöglichen.

\subsection{Fallbeispiel und Diskussion der Ergebnisse}

Für das Fallbeispiel wird ein auf den Vertrieb von Wertpapieren spezialisierter OnlineBroker betrachtet, welcher ein im Vergleich zu einer Universalbank stark eingeschränktes und standardisiertes Produktportfolio anbietet und sich weitgehend auf den Vertriebsweg über Telefon oder Internet konzentriert. Diese geringere Komplexität spiegelt sich in einer sehr standardisierten und integrierten Prozess- sowie Informationssystemlandschaft wider. Gemeinsam mit oftmals vorhandenen schlanken Organisationsstrukturen sind derartige Anbieter bereits heute als stark netzwerkfähig einzustufen.

Der Online-Broker möchte die Wertpapierabwicklung - zunächst für drei Jahre (Betrachtungszeitraum) - an externe Dienstleister abgeben. Es ist zu entscheiden, an wie viele Dienstleister der Auftrag vergeben werden soll. Jeder sei auf Grund der modernen AWSLandschaft mit niedrigen technischen Integrationskosten $k_{\mathrm{T}}$ von 500.000 EUR anzubinden. Gleichzeitig fallen innerhalb des Betrachtungszeitraums nicht-technische Integrationskosten $k_{\mathrm{N}}$ von 100.000 EUR je Dienstleister für die organisatorische Abstimmung und die Koordination an. Es werden einheitliche Service-Level-Verträge geschlossen. Diese sollen garantieren, dass es innerhalb der drei Jahre nur mit einer Wahrscheinlichkeit von 7\% $(w)$

7 Da mehrere lokale Optima auszuschliessen sind, ist die Verstetigung möglich. Die diskrete Lösung $n^{*} \in \mathrm{IN}_{++}$ermittelt sich aus dem stetigen Ergebnis, indem die Gesamtkosten der nächsthöheren bzw. -niedrigeren natürlichen Zahl untersucht werden. Das Ergebnis mit den niedrigeren Gesamtkosten determiniert die optimale diskrete Lösung. Bei identischen Gesamtkosten ist der Entscheider ohne Berücksichtigung weiterer Parameter indifferent. 
zu einem Ausfall kommt ( $w$ ist also zunächst vorgegeben). Der Parameter der Kosten für Ausfallsicherheitsmassnahmen $k_{\mathrm{A}}$ beläuft sich auf 30 Mio. EUR. Die durchschnittliche Ausfalldauer z. B. bei Beschädigung der Datennetze beträgt zwei Tage $(d)$. Pro Tag sind rund 25.000 Transaktionen $(t)$ mit einem durchschnittlichen Volumen von 4.500 EUR $(v)$ abzuwickeln. Die Prozesszeit beträgt drei Tage $(b)$. Der Risikokostensatz wurde mit $15 \%$ $(r)$ kalkuliert.

Die Parameter entsprechen Durchschnittswerten: So ist das 2007 über den Online-Broker Cortal Consors gehandelte Transaktionsvolumen pro Tag vergleichbar (Cortal Consors 2007). Kunden haben mit zwei bis drei Tagen zur endgültigen Abwicklung eines Wertpapiergeschäfts zu rechnen. Ausfallwahrscheinlichkeit und -dauer sind konservativ geschätzt und entsprechen bei 250 Arbeitstagen pro Jahr einer Verfügbarkeit von 99,7 \%.

Für den Online-Broker lässt sich $n^{*}=2$ mit erwarteten Gesamtkosten von 93,546 Mio. EUR bestimmen. Wird nur eine Transaktionsbank integriert, entstehen erwartete Gesamtkosten in Höhe von 95,143 Mio. EUR und ein Nachteil von rund 2\%. Dieses Ergebnis ändert sich kaum, wenn von degressiv steigenden Kosten für die Integration ausgegangen wird. Damit die Anbindung einer dritten Transaktionsbank ökonomisch sinnvoll wird $\left(n^{*}=3\right)$, müssten die Kosten für die Integration c. p. unter 117.000 EUR fallen. Sind die Möglichkeiten zur Risikosenkung bei alleiniger Optimierung der Anzahl anzubindender Transaktionsbanken noch gering, zeigt sich bei gleichzeitiger Anpassung der Ausfallwahrscheinlichkeit ein grösserer Effekt: Der Online Broker bindet $n^{*}=3$ an und wählt mit $w^{*}=0,17$ eine wesentlich geringere Anforderung an die Ausfallsicherheit. Mit Gesamtkosten von 85,240 Mio. EUR ergibt sich ein zusätzlicher Vorteil von rund $9 \%$ gegenüber dem vorher berechneten Optimum mit $n^{*}=2$ und $w^{*}=0,07$. Progressiv steigende Kosten für die Integration der Transaktionsbanken sind wie o. a. vorstellbar und würden tendenziell zu einer Verringerung der optimalen Anzahl führen.

Unter den gegebenen Parameterkonstellationen erscheint somit bei ausreichend niedrigen Integrationskosten die Anbindung mehr als einer Transaktionsbank ökonomisch sinnvoll. Die mit steigender Anzahl zunehmenden Integrationskosten können durch die sinkenden Risikokosten überkompensiert werden. Die optimale Höhe der Ausfallwahrscheinlichkeit bei Anbindung mehr als einer einzigen TB liegt zudem wesentlich über der normalerweise geforderten Ausfallwahrscheinlichkeit.

\subsection{Computergestützte Simulation zur Beantwortung der erweiterten Forschungsfrage}

In der computergestützten Simulation werden zunächst zwei Szenarien verglichen, um die Auswirkung sinkender technischer Integrationskosten zu untersuchen: Im Szenario hoher $k_{\mathrm{T}}$ wird für die gesamten Integrationskosten $\left(k_{\mathrm{T}}+k_{\mathrm{N}}\right)$ ein Intervall von 11 bis 17 Mio. EUR, im Szenario niedriger $k_{\mathrm{T}}$ ein Intervall von 200.000 und 4 Mio. EUR angenommen. Der Kostenparameter $k_{\mathrm{A}}$ wurde in beiden Szenarien auf das Intervall von 20 bis 30 Mio. EUR festgelegt. ${ }^{8}$ Die Simulation umfasst jeweils 1.000 Läufe, bei welchen zunächst per Zufallsoperator die Ausprägungen der Modellparameter aus dem jeweiligen Definitionsbereich bestimmt wurden. Dann wurde die jeweils gesamtkostenminimale Kombination der Optimierungsvariablen $n$ und $w$ über numerische Verfahren ermittelt. Abbildung 1 zeigt deren graphische Aufbereitung. Jeder Punkt markiert die für die jeweilige Umgebung der

8 Die anderen Modellparameter wurden aus dem vorangehenden Abschnitt übernommen (Zeiteinheit: 1 Tag; $b=3 ; d=2 ; r=0,15 ; t=25.000 ; v=4.500)$. 
zufällig bestimmten Modellparameter kostenminimale Kombination. Auf der Ordinate ist die Höhe der Ausfallwahrscheinlichkeit, auf der Abszisse die Anzahl der anzubindenden Transaktionsbanken abgetragen.
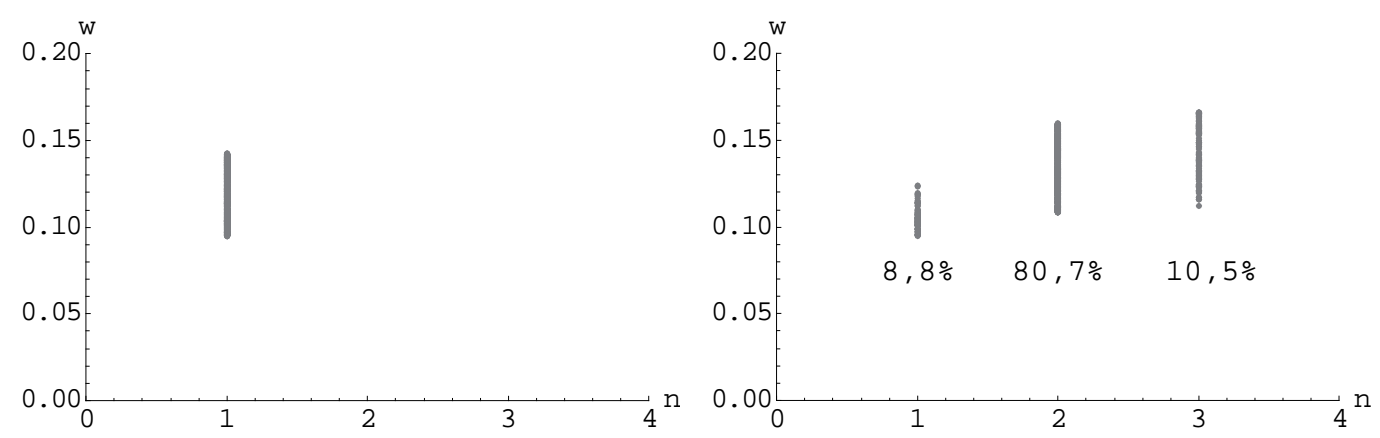

Abbildung 1: Optimale Kombination $\left(n^{*} ; w^{*}\right)$ aus 1.000 Simulationsläufen bei hohen (links) und niedrigen (rechts) Integrationskosten

Die Grafiken zeigen, dass bei niedrigen $k_{\mathrm{T}}$ mehr als eine TB anzubinden ist, wobei sich die optimalen Werte für $n$ wie erwartet im niedrigen einstelligen Bereich bewegen. Gerade der Schritt von einer auf zwei Transaktionsbanken verringert die Risikokosten wesentlich, da damit überhaupt erst die Möglichkeit geschaffen wird, Transaktionen umzuleiten. ${ }^{9}$

Unter Berücksichtigung der beschriebenen Modernisierungsaktivitäten der FDL und der damit zu erwartenden stark sinkenden Integrationskosten zeigt die Simulation, dass in mehr als $80 \%$ der Fälle mehr als eine Transaktionsbank kostenminimal ist. Bei rd. 10\% sind sogar mehr als zwei Transaktionsbanken anzubinden, wobei die optimale Ausfallwahrscheinlichkeit für ein Optimum bei minimalen Gesamtkosten dann entsprechend höher zu wählen ist.

Die Anbindung von mehr als zwei Transaktionsbanken erscheint vor dem Hintergrund der derzeitigen Praxis zunächst ungewöhnlich. Die Ergebnisse sind bei genauerer Betrachtung jedoch durchaus plausibel. Die eigentliche Hürde der praktischen Umsetzung stellen ausreichend standardisierte und flexibilisierte AWS sowie organisatorische Standards zum Management der Dienstleistungsbeziehung (z. B. ITIL) dar, mit welchen die Anbindung der Dienstleister auf der Basis allgemein anerkannter Standards vorgenommen werden kann und proprietäre Anpassungen auf einen bestimmten Dienstleister vermieden werden können. Ist dieser Grundstein gelegt, dürfte auch die Einbindung weiterer Dienstleister technisch und organisatorisch abbildbar sein.

Vergleicht man im Szenario niedriger $k_{\mathrm{T}}$ die optimalen Kombinationen mit der jeweils höchsten bzw. niedrigsten Ausfallwahrscheinlichkeit in Abhängigkeit der Anzahl an Transaktionsbanken, wird deutlich, dass die Anforderungen an die Ausfallsicherheit mit zunehmender Anzahl Transaktionsbanken sinken. Werden einzelne Modellparameter zur Sensitivitätsanalyse variiert, zeigen sich gerade bei der Höhe des je Zeiteinheit abzuwi-

9 Der breite Korridor zwischen min. und max. Ausfallwahrscheinlichkeit bei einer gegebenen Anzahl Transaktionsbanken erklärt sich aus dem breiten Definitionsintervall von $k_{\mathrm{A}}$. Je nach Höhe dieser Kosten können bei Verringerung der Ausfallwahrscheinlichkeit verschieden hohe Beträge zur Kompensation steigender Risikokosten freigesetzt werden. 
ckelnden Transaktionsvolumens interessante Ergebnisse. Die graphische Aufbereitung der 1.000 Simulationsläufe mit niedrigem bzw. hohem Transaktionsvolumen zeigt Abbildung 2. Bei alleiniger Optimierung nach $n$ gingen niedrige Transaktionsvolumina mit einer niedrigen Anzahl anzubindender Transaktionsbanken einher. Nun werden bei niedrigem Transaktionsvolumen tendenziell mehr Transaktionsbanken angebunden und es wird gleichzeitig eine wesentlich höhere Ausfallwahrscheinlichkeit zugelassen. Dies erklärt sich über den unterschiedlichen Einfluss der beiden Optimierungsparameter auf die Risikokosten sowie der linearen Abhängigkeit vom abzuwickelnden Transaktionsvolumen.
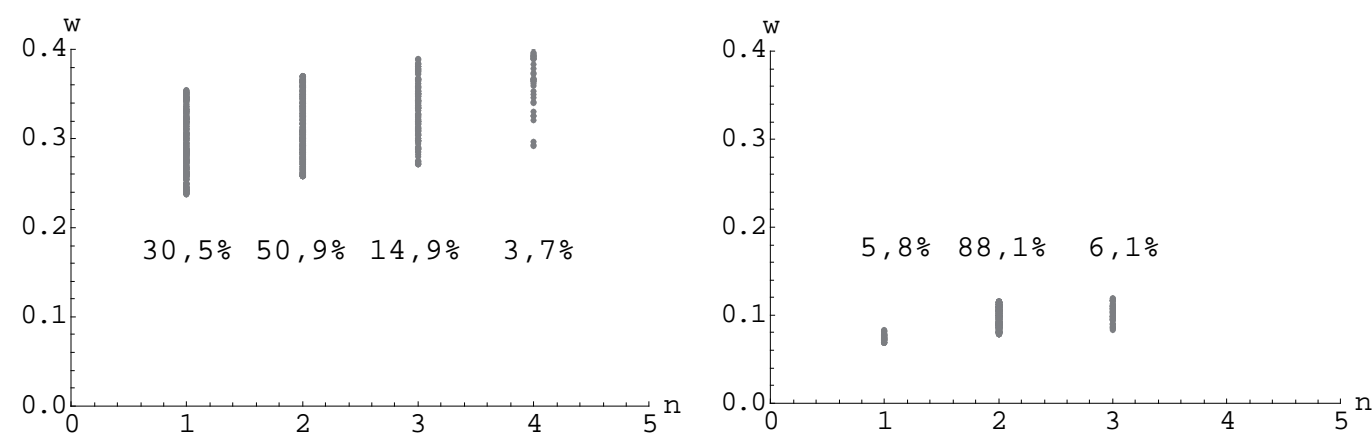

Abbildung 2: Optimale Kombination aus 1.000 Simulationsläufen bei niedrigem bzw. hohem (10.000 bzw. 35.000 Transaktionen zu je 4.500 EUR) Transaktionsvolumen.

\subsection{Einfluss von Abwicklungskosten und Kosten zur Umleitung von Transaktionen}

Das Modell bildet Abwicklungskosten, welche für jede abzuwickelnde Transaktion zu entrichten sind, implizit als linear zur Anzahl Transaktionen und über alle Transaktionsbanken identisch ab. Berücksichtigt man hingegen die in der Praxis zahlreich vorhandenen Preismodelle z. B. mit Mengenrabatten, Fixbeträgen oder Stufenmodellen, tritt bei der Entscheidung über die Anzahl anzubindender Transaktionsbanken ein weiterer, zu berücksichtigender Trade-Off hinzu.

Die Preismodelle definieren direkt (Mengenrabatt) bzw. indirekt (Fixbetrag) Mengengrenzen, ab welchen die durchschnittlichen Abwicklungskosten je Transaktion sinken. Für die Entscheidung über die Anzahl anzubindender Transaktionsbanken folgt daraus, dass bei Hinzunahme jeder weiteren Transaktionsbank (Grenzbetrachtung) geprüft werden muss, ob Mengengrenzen bei der initialen Aufteilung bzw. Umleitung der Transaktionen im Falle eines Ausfalls (die Anzahl umzuleitender Transaktionen determinieren die dafür anfallenden Abwicklungskosten) immer noch erreicht werden können oder mit höheren Abwicklungskosten zu rechnen ist. Eine Differenz muss dann analog den mit jeder hinzukommenden Transaktionsbank zusätzlich anfallenden Integrationskosten durch sinkende Risikokosten kompensiert werden können. Tendenziell wird so die Anzahl anzubindender Transaktionsbanken geringer ausfallen als ohne Berücksichtigung der Abwicklungskosten.

Bezogen auf die Fallstudie, in welcher zunächst ein Gesamtkostenvorteil von 1,597 Mio. EUR bei der Anbindung von zwei Transaktionsbanken erreicht wurde, ist also zu prüfen, ob dieser Betrag ggf. anfallende höhere Abwicklungskosten aus der Aufteilung der abzuwickelnden Transaktionen kompensiert. 
Ein derartiges zweistufiges Vorgehen erscheint auf Grund der Vielzahl bestehender Preismodelle, zudem in Kombination, wenn die in Frage kommenden Transaktionsbanken mit verschiedenen Preismodellen arbeiten, auch für den Einsatz in der Praxis geeignet. Auf diese Weise lassen sich zudem aus der durch das Modell ermittelten Anzahl anzubindender Dienstleister und der damit einhergehenden Aufteilung der Transaktionen Ansatzpunkte für die Verhandlung der Abwicklungspreise bei der Vertragsgestaltung erkennen.

Des Weiteren sind die Wechselkosten - im Sinne der mit einer Umleitung von Transaktionen verbundenen Kosten - im Modell vereinfachend als vernachlässigbar angenommen, da Erfahrungswerte für eine sinnvolle Abschätzung dieser Kosten nicht vorhanden sind. Trotz hoher Automatisierung der Wertpapierabwicklung kann die bei einem Ausfall notwendige Umleitung aber unter Umständen eine kostenintensive manuelle Bearbeitung und damit auch eine Verzögerung der Abwicklung nach sich ziehen. Zudem ist es denkbar, dass kurzfristig angeforderte Kapazitäten bei den vom Ausfall nicht betroffenen Transaktionsbanken mit höheren Abwicklungspreisen zu vergüten sind. Die Kosten zur Umleitung steigen demnach mit den bei einem Ausfall jeweils umzuleitenden Transaktionen.

Das Modell müsste zur Abbildung derartiger Kosten angepasst werden. Ausgangspunkt könnte hierfür eine Erweiterung des Risikoterms zur Modellierung zusätzlicher Verzögerungen bzw. die Einführung eines zusätzlichen Kostenterms sein. Mit Letzterem könnten einerseits variable Kostenbestandteile, welche mit der Anzahl umzuleitender Transaktionen steigen (z. B. Aufschläge bei Abwicklungspreisen oder manuelle Nachbearbeitung) berücksichtigt werden. Andererseits könnten darin auch fixe Kostenbestandteile, die je Ausfall etwa in gleicher Höhe anfallen (z. B. Identifikation betroffener Transaktionen, Anfordern zusätzlicher Verarbeitungskapazität, Vermeidung von Doppelabwicklungen) integriert werden.

Grundsätzlich stellen derartige Kosten ein Hemmnis dar, überhaupt auf eine MultipleSourcing-Strategie zu wechseln. Denn erst bei mehr als einer angebundenen Transaktionsbank ist eine Umleitung möglich. Fixe Kostenbestandteile je Ausfall gewinnen zudem mit steigender Anzahl an Transaktionsbanken an Bedeutung, da mit der Anzahl an Transaktionsbanken auch die Anzahl der zu erwartenden Ausfälle steigt. Multiple-Sourcing mit vielen angebundenen Transaktionsbanken wird dadurch tendenziell weniger attraktiv.

Die Wechselkosten sind gerade auch vor dem Hintergrund der Ergebnisse der erweiterten Forschungsfrage interessant. So führt eine Berücksichtigung dieser Kosten c. p. dazu, dass die Anforderungen an die Verfügbarkeit der angebundenen Dienstleister tendenziell höher ausfallen werden. Dies ist einleuchtend, da die Notwendigkeit einer Umleitung direkt mit der Verfügbarkeit der Dienstleister zusammenhängt.

\subsection{Limitationen des Modells und weiterer Forschungsbedarf}

Die Entscheidung über den Einsatz einer Multiple-Sourcing-Strategie wird im vorliegenden Optimierungsmodell rein kostenbasiert getroffen. Durch fehlende Differenzierungsoptionen hin zum Kunden, die geringen Margen und dem dennoch damit verbundenen hohen operationellen Risiko ist diese Vorgehensweise für den betrachteten Bereich gerechtfertigt. Die dabei für eine allgemeine Modellbildung notwendigen Vereinfachungen werden im Folgenden mit ihren Auswirkungen auf die Ergebnisse und dem daraus resultierenden weiteren Forschungsbedarf diskutiert:

In den Integrationskosten wurden die Kosten für die einmalige technische und organisatorische Integration sowie laufende Kosten für das Management der Dienstleister zusam- 
mengefasst und insgesamt ein linearer Verlauf unterstellt. Die Diskussion des Fallbeispiels lässt zwar eine gewisse Robustheit der Ergebnisse auch gegenüber anderen Kostenverläufen vermuten, in weiterführenden Untersuchungen wäre dennoch eine detailliertere Analyse der verschiedenen Kostenbestandteile und deren Verlauf bei Anbindung mehr als einer Transaktionsbank notwendig.

Bei der Ermittlung der Risikokosten wurde implizit unterstellt, dass es sich um einen risikoneutralen Entscheider handelt. Auch wurde ein linearer Zusammenhang mit der Länge eines Ausfalls angenommen. Würden auch indirekte Effekte eines Ausfalls, wie z. B. ein zusätzlicher Vertrauensverlust beachtet, ist ggf. von überproportional mit der Ausfalldauer steigenden Risikokosten auszugehen. Ebenso wurden Risikokosten eines Ausfalls, welcher durch Ausfallsicherheitsmassnahmen nicht beeinflusst werden kann (z. B. durch Insolvenz) nicht betrachtet. Die Risikokosten im Modell sind also tendenziell zu niedrig angesetzt. Höhere Risikokosten verstärken jedoch die aus der Modellanalyse gewonnene Aussage, dass die Anbindung mehr als eines Dienstleisters ökonomisch sinnvoll sein kann.

Hinsichtlich der im vorangehenden Abschnitt diskutierten Abwicklungskosten ist eine detaillierte Analyse der einzelnen Preismodelle als Teil weiterführender Forschungsarbeiten sinnvoll und wichtig. Aus technischer Sicht ist es notwendig, die zur Umleitung von Transaktionen erforderlichen Mechanismen zu identifizieren sowie zur exakten Bestimmung dabei anfallender Kosten die damit zusammenhängenden zeitlichen und organisatorischen Effekte zu analysieren. Auch hinsichtlich einer effizienten Kapazitätsplanung auf Seiten der Dienstleister sind die Effekte von Multiple-Sourcing und der damit zusammenhängenden, oftmals kurzfristigen Anforderung an Verarbeitungskapazität beim Umleiten von Transaktionen ein sinnvoller Teil weiterer Forschungsarbeit.

\section{Zusammenfassung und Gestaltungsempfehlungen}

Vor dem Hintergrund sinkender Integrationskosten wurde in diesem Beitrag untersucht, ob Multiple-Sourcing zur Risikosteuerung in Wertschöpfungsnetzen von FDL ökonomisch sinnvoll sein kann. Am Beispiel von Wertpapiertransaktionsbanken zeigt sich mit Hilfe eines Optimierungsmodells, dass insbesondere bei hohen Risikokosten die Anbindung mehr als eines Dienstleisters vorteilhaft sein kann. Die Höhe der Risikokosten hängt dabei wesentlich vom gehandelten Transaktionsvolumen, aber auch dem Risikokostensatz ab. Letzterer beinhaltet $u$. a. notwendige Kosten zur Zwischenfinanzierung nicht abgewickelter Transaktionen. Gerade in Zeiten hochvolatiler Märkte und schwieriger Finanzierungsbedingungen, wie während der immer noch nachwirkenden Finanzmarktkrise, bietet sich Multiple-Sourcing zur Verringerung der hier diskutierten Risikokosten an.

Davon ausgehend wird weiter untersucht, wie sich eine Multiple-Sourcing-Strategie auf die Anforderungen an die Verfügbarkeit der technischen Systeme und damit auf die Summe aus Risikokosten eines Ausfalls und den Kosten für Ausfallsicherheitsmassnahmen als relevante Entscheidungsgrösse auswirkt. Werden nämlich Ausfallrisiken durch das redundante Vorhalten von Infrastruktur und Personal zu minimieren versucht, führt dies zu extrem hohen Fixkosten. Mit einer Simulation konnte gezeigt werden, dass das Ausfallrisiko auch durch die Anbindung mehrerer Wertschöpfungspartner reduziert und so auf hohe, mit zunehmenden Verfügbarkeitsanforderungen progressiv steigende Kosten für Ausfallsicherheitsmassnahmen verzichtet werden kann. Die Simulation zeigt, dass sich ein Gesamtkostenminimum bei zwei oder mehr Transaktionsbanken und entsprechend reduzierter Ausfallsicherheit einstellt. 
Die Erkenntnisse über die zu wählende Anzahl an Dienstleistern aus diesem Beitrag stützen die Ergebnisse vergleichbarer Forschungsarbeiten, auch aus anderen Branchen, in welchen der Trade-Off zwischen Transaktionskosten und Risikokosten eines Ausfalls unter gleichen Voraussetzungen betrachtet werden. Auch dort wird Multiple-Sourcing nur bei höheren Ausfallwahrscheinlichkeiten der einzelnen Dienstleister bzw. verallgemeinernd höheren Risikokosten (z. B. bei zeitkritischen Dienstleisterbeziehungen der Just-in-timeProduktion) empfohlen. Die optimale Anzahl bewegt sich meist im unteren einstelligen Bereich. Sehr grosse Dienstleisterpools werden auf Grund der dann stark steigenden Komplexität und der intensiven Abstimmungsnotwendigkeit nicht empfohlen. Dabei werden die Ausfallwahrscheinlichkeiten der einzelnen Dienstleister stets als exogene Grösse angenommen. Keine der bekannten Untersuchungen beschäftigen sich im Kontext von MultipleSourcing explizit mit der Fragestellung nach einer Optimierung der Anzahl und gleichzeitig der Zuverlässigkeit der Dienstleister, wie sie das Modell zur Beantwortung der erweiterten Forschungsfrage abbildet.

Verallgemeinernd lassen sich folgende Schlussfolgerungen ziehen:

- Die bisherige Praxis der Branche, nur einen einzigen Dienstleister für bestimmte Aufgaben heranzuziehen, kann unter der Voraussetzung sinkender technischer Integrationskosten in bestimmten Anwendungsfällen auch über die Wertpapierabwicklung hinaus als überholt angesehen werden. Es muss im Einzelfall geprüft werden, welche SourcingStrategie zu einer optimalen Lösung führt. Die damit verbundene mögliche Reduzierung von Kosten, auch hinsichtlich der Verpflichtung, das operationelle Risiko mit regulatorischem Eigenkapital zu unterlegen, rechtfertigen die dazu notwendigen Prüfungen.

- Durch die Anbindung mehr als eines Dienstleisters ergibt sich eine weitere Option zur Optimierung der Dienstleisterbeziehung: Die Anforderungen an die Ausfallsicherheit können abgeschwächt werden, da die ökonomisch sinnvolle Ausfallsicherheit durch die Steigerung der Dienstleisteranzahl relaxiert und die Robustheit des Zusammenwirkens der Unternehmen auf diese Weise gestärkt wird. So können Investitionen in Ausfallsicherheitsmassnahmen reduziert werden, was letztlich zu günstigeren Preisen der ausgelagerten Dienstleistung und einem Kostenvorteil für den auslagernden FDL führt.

Die in der Simulation gefundenen Ergebnisse deuten darauf hin, dass durch geeignet gestaltete Preismodelle in Zusammenspiel mit Multiple-Sourcing auf Seiten der FDL auch für die Dienstleister Gestaltungsoptionen zur Produkt- bzw. Preisdifferenzierung eröffnet werden. Anstatt Abwicklungsleistungen wie bisher mit weitgehend identischer Qualität (Commodity-Produkt) anzubieten, werden ggf. auch Angebote mit geringerer Ausfallsicherheit zu günstigeren Abwicklungspreisen attraktiv (siehe die allgemeine Diskussion bei Pan 2009).

\section{Anhang}

\section{Herleitung des Risikokostenterms (3)}

Zwei Fälle sind zur Ermittlung der Risikokosten zu unterscheiden: Fallen $i \leq n-1$ der angebundenen $n$ TB aus, betrifft dies nur die Transaktionen in Abwicklung. Da bei jeder angebundenen TB zu jeder Zeit beliebig viele Transaktionen abgewickelt werden können, können während des Ausfalls hinzukommende Transaktionen von den nicht ausgefallenen TB ohne Verzögerung abgewickelt werden. Zu jedem Zeitpunkt sind bei jeder TB $b \cdot t / n$ TA in Abwicklung, bei $i \leq n-1$ ausfallenden TB also insgesamt $i \cdot b \cdot t / n$ Transaktionen. 
Da diese gleichmässig über die Zeit verteilt eintreffen, befindet sich zum Zeitpunkt des Ausfalls jede Einzelne bereits durchschnittlich (da die Risikokosten proportional hinsichtlich des verzögerten Transaktionsvolumens wie auch der Verzögerungsdauer steigen, kann mit Durchschnittswerten gerechnet werden) $b / 2$ Zeiteinheiten im Abwicklungsprozess. So verzögert sich durch die erneute Abwicklung dieser Transaktionen nach dem Ausfall die endgültige Abwicklung um durchschnittlich $d+b / 2$ Zeiteinheiten. Der im Betrachtungszeitrum einmalige und gleichzeitige Ausfall von i der $n-1$ TB tritt mit einer Wahrscheinlichkeit von $\left(\begin{array}{l}n \\ i\end{array}\right) \cdot w^{i} \cdot(1-w)^{n-i}$ auf, so dass sich erwartete Risikokosten i. H. v.

$$
\sum_{i=1}^{n-1}\left[\left(\begin{array}{l}
n \\
i
\end{array}\right) \cdot w^{i} \cdot(1-w)^{n-i} \cdot i \cdot b \cdot \frac{t}{n} \cdot\left(d+\frac{b}{2}\right) \cdot v \cdot r\right]
$$

ergeben. Ist nur eine einzige TB angebunden, ist eine Abwicklung der Transaktionen bei einem Ausfall generell unmöglich. Auch dieser Fall wird durch die Formel abgebildet. Bei $n=1$ ist die Bedingung des Summenzeichens nie erfüllt.

Fallen dagegen alle $n$ angebundenen TB gleichzeitig aus, sind $n \cdot b \cdot t / n$ Transaktionen in Abwicklung betroffen. Zudem verzögert sich nun auch die Abwicklung der während des Ausfalls der Länge $d$ neu hinzukommenden $d \cdot n \cdot t / n$ Transaktionen bis zum Ende des Ausfalls. Diese treffen wiederum gleichmässig über die Zeit verteilt bei den TB ein, so dass sich eine durchschnittliche Verzögerung von $d / 2$ ergibt. Es fallen alle $n$ angebundenen TB gemeinsam im Betrachtungszeitraum mit einer Wahrscheinlichkeit von $w^{\text {n }}$ aus. Für diesen Fall ergeben sich nach Annahme (A4) erwartete Risikokosten in Höhe von

$$
w^{n} \cdot\left[b \cdot t \cdot\left(d+\frac{b}{2}\right)+d \cdot t \cdot \frac{d}{2}\right] \cdot v \cdot r .
$$

Die Summe der Risikokosten beider Fälle lässt sich umformen zu

$$
\begin{aligned}
& K_{R}(n)=b \cdot t \cdot\left(d+\frac{b}{2}\right) \cdot v \cdot r \cdot\left\{\sum_{i=0}^{n}\left[\left(\begin{array}{l}
n \\
i
\end{array}\right) \cdot w^{i} \cdot(1-w)^{n-i} \cdot \frac{i}{n}\right]-w^{n}\right\} \\
& +w^{n}\left[b \cdot t \cdot\left(d+\frac{b}{2}\right)+d \cdot t \cdot \frac{d}{2}\right] \cdot v \cdot r,
\end{aligned}
$$

wobei die Änderung von Unter- und Obergrenze der Summe zu beachten ist. Einsetzen und Ausklammern von $w$ führt im Summenterm von Formel (7) zu

$$
\sum_{i=0}^{n}\left(\begin{array}{l}
n \\
i
\end{array}\right) \cdot w^{i} \cdot(1-w)^{n-i} \cdot \frac{i}{n}=w \cdot\left[\begin{array}{l}
n \cdot(1-w)^{n-1} \cdot \frac{1}{n}+\frac{n \cdot(n-1)}{2 !} \cdot w^{1} \cdot(1-w)^{n-2} \cdot \frac{2}{n}+\ldots+ \\
+n \cdot w^{n-2} \cdot(1-w)^{1} \cdot \frac{n-1}{n}+1 \cdot w^{n-1} \cdot \frac{n}{n}
\end{array}\right] .
$$

Erweitern mit $w+(1-w) / w+(1-w)$ führt zu

$$
\sum_{i=0}^{n}\left(\begin{array}{l}
n \\
i
\end{array}\right) \cdot w^{i} \cdot(1-w)^{n-i} \cdot \frac{i}{n}=w \cdot \frac{\sum_{i=0}^{n}\left(\begin{array}{l}
n \\
i
\end{array}\right) \cdot w^{i} \cdot(1-w)^{n-i}}{w+(1-w)} .
$$

Die Summe im Zähler des Bruches der rechten Seite der Gleichung kann substituiert werden durch $[w+(1-w)]^{n}$ : 


$$
\sum_{i=0}^{n}\left(\begin{array}{l}
n \\
i
\end{array}\right) \cdot w^{i} \cdot(1-w)^{n-i} \cdot \frac{i}{n}=w \cdot \frac{[w+(1-w)]^{n}}{w+(1-w)}=w .
$$

Eingesetzt in (7), Ausklammern von $t \cdot v \cdot r$ und Ausmultiplizieren führt zu Formel (3).

\section{Untersuchung der Gesamtkostenfunktion bei Formel (6)}

Die erste Ableitung der Gesamtkostenfunktion ergibt

$$
\frac{\partial K_{G}(\dot{n})}{\partial \dot{n}}=\left(k_{T}+k_{N}\right)+t \cdot \frac{d^{2}}{2} \cdot w^{\dot{n}} \cdot \ln w \cdot v \cdot r .
$$

Wie unter Berücksichtigung von $\ln w<0 \forall w \in] 0 ; 1[$ anhand der Grenzwerte

$$
\lim _{\dot{n} \rightarrow-\infty} \frac{\partial K_{G}(\dot{n})}{\partial \dot{n}} \rightarrow-\infty<0 \text { und } \lim _{\dot{n} \rightarrow \infty} \frac{\partial K_{G}(\dot{n})}{\partial \dot{n}}=k_{I}>0
$$

ersichtlich ist, besitzt sie mindestens eine Nullstelle in $\dot{n}$. Die in IR stets positive zweite Ableitung von

$$
\frac{\partial^{2} K_{G}(\dot{n})}{\partial \dot{n}^{2}}=t \cdot \frac{d^{2}}{2} \cdot w^{\dot{n}} \cdot(\ln w)^{2} \cdot v \cdot r>0 \forall \dot{n}
$$

zeigt weiter, dass die erste Ableitung streng monoton steigt, also genau eine Nullstelle besitzt.

\section{Literaturhinweise}

Alt, R./Fleisch, E. (2002): Netzwerkfähigkeit von Unternehmen, in: Österle, H./Fleisch, E./ Alt, R. (Hrsg.): Business Networking in der Praxis, 2. Aufl., Berlin, S. 353-369.

Alt, R./Zerndt, T. (2008): Finanznetzwerke durch Outsourcing - das Beispiel der Schweiz, in: Kaib, B. (Hrsg.): Outsourcing in Banken, Wiesbaden, S. 315-343.

Alt, R./Zerndt, T. (2009): Transformation durch Sourcing bei Banken, in: Alt, R./Bernet, B./Zerndt, T. (Hrsg.): Transformation von Banken, Berlin, S. 4-20.

Alt, R. (2010): Wir müssen Bank-IT-Anwendungen mehr vom Kunden her denken, in: Wirtschaftsinformatik \& Management, Jg. 2, Nr. 4, S. 6-11.

Alt, R./Puschmann, T./Koblmann, F./Zerndt, T. (2010): Eckpunkte für die Universalbank 2015, in: Die Bank, Nr. 3, S. 40-44.

Becker, A./Buxmann, P./Widjaja, T. (2009): Value potential and challenges of service-oriented architectures - A user and vendor perspective, in: Newell, S./Whitley, E./Pouloudi, N./Wareham, J./Mathiassen, L. (Hrsg.): Proceedings of the 17th European Conference on Information Systems, ECIS, Verona, June 2009, S. 2085-2096.

Berger, P./Gerstenfeld, A./Zeng, A. (2004): How many suppliers are best? A decision-analysis approach, in: Omega, Jg. 32, S. 9-15.

Berger, P./Zeng, A. (2006): Single versus multiple sourcing in the presence of risk, in: Journal of the Operational Research Society, Jg. 57, Nr. 3, S. 250-261. 
Burke, G./Carillo J./Vakharia A. (2007): Single versus multiple supplier sourcing strategies, in: European Journal of Operational Research, Jg. 182, S. 95-112.

Cortal Consors (2007): Cortal Consors Unternehmensbroschüre 2007, unter https://www.cortalconsors.de/euroWebDe/servlets/embeddedSourcesServlet?contentType=pdf\&contentExtId=denps31349977\&iframe=yes, Abfrage am 1.3.2011.

Craighead, C./Blackhurst, J./Rungtusanatham, M./Handfield, R. (2007): The Severity of Supply Chain Disruptions: Design Characteristics and Mitigation Capabilities, in: Decision Sciences, Jg. 38, S. 131-156.

Erl, T. (2007): SOA Principles of Service Design, Boston.

Falkenberg, F./Müller, R./Bönsch, J. (2006): Wertschöpfungsmodelle der Zukunft - Banken und Provider 2010, Studie der Universität St. Gallen, St. Gallen.

Friedrich, D. (2006): Banken hoffen auf SOA bei Kernbank-Systemen, unter http://www.cio.de/ knowledgecenter/erp/812858, Abfrage am 1.3.2011.

Harishankar, R. (2009): SOA-Based Enterprise Integration, New York.

Homburg, C. (1995): Single Sourcing, Double Sourcing, Multiple Sourcing...?, in: Zeitschrift für Betriebswirtschaft, Jg. 65, S. 813-834.

Joiko, M. (2006): Modernisierung der IT-Landschaft: Fortschritt durch Fusionen, in: Die Bank, Nr. 5, S. 68-71.

Kohlmann, F./Alt, R. (2010): The Impact of Service-oriented Architecture on Business Networkability, in: Alexander, T./Turpin, M./van Deventer, J.P. (Hrsg.): Proceedings of the 18th European Conference on Information Systems, ECIS, Pretoria, June 2010.

Kröpfl, S. (2003): Effizienz in der Abwicklung von Wertpapiergeschäften. Transaktionskosten und Wettbewerb in Europa, Berlin.

Linthicum, D. (2003): Enterprise Application Integration, 4. Aufl., Boston.

Mansfeld, K./Alt, R./Puschmann, T. (2010): Integrierte Gestaltung und Bewertung zur Entscheidungsunterstützung im Financial Sourcing, in: Banking and Information Technology, Nr. 2, S. 45-55.

Pan, W. (2009): Dynamic Pricing Strategy of Provider with Different QoS Levels in Web Service, in: Journal of Networks, Jg. 4, Nr. 4, S. 228-235.

Quack, K. (2010): IT-Strategien: Deutsche Bank vertraut im Core-Banking SAP, in Computerwoche, Nr. 5, S. 34.

Regniet, S. (2007): Banken, Vista und BPO treiben den stagnierenden Outsourcing-Markt an, in: Netzwoche, Nr. 5, S. 15-17.

Ruiz-Torres, A./Mahmoodi, F. (2007): The optimal number of suppliers considering the costs of individual supplier failures, in: Omega, Jg. 35, S. 104-115.

Ruh, W./Maginnis, F./Brown, W. (2001): Enterprise Application Integration, New York.

Spabr, R. (2001): Steuerung operationeller Risiken im Electronic und Investment Banking, in: Die Bank, Nr. 9, S. 660-663.

Sterling Commerce (2010): Komplexe IT-Infrastrukturen verursachen bei Banken hohe Kosten für den Datenaustausch, Pressemitteilung zur Studie „No bank is an island” (2010), unter http:// www.sterlingcommerce.com/de/about/news/press-releases/PM_Sibos_StudieDatenaustausch_DE1 0.htm, Abfrage am 1.3.2011.

Wang, D./Zhao, Y. (2007): Decision-making for the Optimal Number of Suppliers Considering Trade-off between Supplier Management Cost and Supply Failure Risk, in: Proceedings of the 
2007 IEEE International Conference on Industrial Engineering and Engineering Management, Singapur, S. 1664-1668.

Weitzel, T./Martin, S./König, W. (2003): Straight Through Processing auf XML-Basis im Wertpapiergeschäft, in: Wirtschaftsinformatik, Jg. 45, Nr. 4, S. 409-420.

Wortmann, F. (2006): Integrationsinfrastrukturen in der Finanzdienstleistungsbranche: Ergebnisse einer Studie, in: Schelp, J./Winter, R. (Hrsg.): Integrationsmanagement, Berlin, S. 169-202.

Hans Ulrich Buhl, Prof. Dr., ist Inhaber des Lehrstuhls für BWL, Wirtschaftsinformatik, Informations- \& Finanzmanagement und Wissenschaftlicher Leiter des Kernkompetenzzentrums Finanz- \& Informationsmanagement, Universität Augsburg.

Christoph Dorsch, Dipl.-Kfm., ist wissenschaftlicher Mitarbeiter am Lehrstuhl für BWL, Wirtschaftsinformatik, Informations- \& Finanzmanagement am Kernkompetenzzentrum Finanz- \& Informationsmanagement.

Matthias Henneberger, Dr., ist Consultant Manager bei der Firma Atos.

Anschrift: FIM Kernkompetenzzentrum, Universität Augsburg, D-86135 Augsburg, Tel.: +49 (0)821/598-4800, Fax: +49 (0)821/598-4899, E-Mail: Hans-Ulrich.Buhl@wiwi.uniaugsburg.de, URL: www.fim-online.eu

Die Autoren danken den anonymen Gutachtern für ihre wertvollen Hinweise. 